\title{
The flared inner disk of the Herbig Ae star AB Aurigae revealed by VLTI/MIDI in the $\mathbf{N}$-band ${ }^{\star}$
}

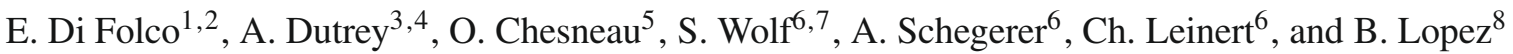 \\ 1 Observatoire de Genève, Université de Genève, Chemin des Maillettes 51, 1290 Sauverny, Switzerland \\ ${ }^{2}$ LESIA, Observatoire de Paris, CNRS-UMR-8109, UPMC, Université Paris Diderot, 5 place J. Janssen, 92195 Meudon, France \\ e-mail: emmanuel.difolco@obspm.fr \\ 3 Université Bordeaux 1; Laboratoire d'Astrophysique de Bordeaux (LAB), France \\ ${ }^{4}$ CNRS/INSU - UMR 5804; BP 89, 33270 Floirac, France \\ e-mail: dutrey@obs.u-bordeaux1.fr \\ 5 UMR 6525 H. Fizeau, Univ. Nice Sophia Antipolis, CNRS, Observatoire de la Côte d'Azur, Av. Copernic, 06130 Grasse, France \\ ${ }^{6}$ Max-Planck Institut für Astronomie, Königstuhl 17, 69117 Heidelberg, Germany \\ 7 University of Kiel, Institute of Theoretical Physics and Astrophysics, Leibnizstrasse 15, 24098 Kiel, Germany \\ 8 Univ. Nice Sophia Antipolis \& Obs. de la Côte d'Azur, Laboratoire H. Fizeau, UMR 6525, BP 4229, 06034 Nice Cedex 4, France
}

Received 4 April 2008 / Accepted 2 February 2009

\section{ABSTRACT}

\begin{abstract}
Aims. We aim at using the long baselines of the VLT Interferometer and the mid-IR combiner MIDI ( $8-13 \mu$ m) to derive the morphology of the protoplanetary disk surrounding the Herbig Ae star AB Aurigae

Methods. We present the first $N$-band analysis of $\mathrm{AB}$ Aur performed with a maximum angular resolution of $17 \mathrm{mas}(2.5 \mathrm{AU}$ at the Taurus-Auriga distance). We used the radiative transfer code MC3D and a silicate-dominated dust grain mixture to fit the spectral energy distribution (SED), together with the $N$-band dispersed visibilities $(\lambda / \delta \lambda \sim 30)$ and to constrain the inner-disk spatial structure. Results. The silicate band is prominent in the $\sim 300$ mas FOV of the MIDI instrument, the emission reaches 70 to $90 \%$ of the total flux measured by ISO. The circumstellar emission (CSE) is resolved even at the shortest baselines. The spectrally dispersed visibilities show a steep drop between 8 and $9.5 \mu \mathrm{m}$, followed by a plateau between 10 and $13 \mu \mathrm{m}$. Our modelling shows that the observed SED and visibilities can be reproduced with a simple passive disk model. For such a weakly inclined disk $(i \sim 30$ deg $)$, the mid-IR visibilities can directly determine the flaring index, while the scale height can be subsequently and unambiguously derived from the combination of the spectral and interferometric constraints. The modelling yields typical values for the scale height of about 8 AU at a radial distance of $100 \mathrm{AU}$ and a flaring index in the range 1.25-1.30 for the explored range of model input parameters.

Conclusions. The radial structure of the circumstellar inner disk around AB Aur is directly determined by MIDI. The radiative transfer modelling demonstrates the powerful synergy of interferometry and spectro-photometry to alleviate the degeneracy, which may hamper determining the disk morphology. Our analysis supports the classification of AB Aur among the flared disks of the first group
\end{abstract} in the Meeus classification.

Key words. stars: individual: AB Aur - stars: circumstellar matter - stars: pre-main sequence - methods: observational techniques: interferometric

\section{Introduction}

With a luminosity of the order of $47 L_{\odot}$ and a distance of $144 \pm_{17}^{23}$ pc (van den Ancker et al. 1998), AB Aurigae is one of the nearest, brightest, and best-studied Herbig Ae stars of spectral type A0-A1 (Hernández et al. 2004). In the classification of the Herbig Ae circumstellar matter, the modelling of the SED in the Mid-IR provided by the analysis of the ISO data (Meeus et al. 2001; Bouwman et al. 2000) shows that AB Aurigae belongs to Group I, since the young star appears surrounded by a flaring disk responsible for the bump in the mid-IR.

An outer disk (surrounded by a large envelope) is observed at all wavelengths. NIR imaging with the HST-STIS Grady et al. (1999) reveals a flattened reflection nebula, close to pole-on, which extends up to $r \sim 1300 \mathrm{AU}$ from the star. Using the Subaru telescope and its Coronographic Imager and Adaptive Optics systems, Fukagawa et al. (2004) show that the circumstellar matter in the outer disk presents a spiral structure, also

* Based on observations collected at ESO (Paranal Observatory) with the VLT Interferometer - Prog ID: 074.C-552 \& 076.C-252. observed at millimetre ( $\mathrm{mm}$ ) wavelengths by Piétu et al. (2005) and Lin et al. (2006). Images of the thermal dust emission at 11 and $18 \mu \mathrm{m}$ obtained by Mariñas et al. (2006) using Michelle on Gemini North reveal that the bulk of the dust emission has a size of about $20 \mathrm{AU}$, but a faint dust component also extends up to 280-350 AU, in agreement with the size derived from the mm continuum observations (Piétu et al. 2005). The CO line kinematics also reveal that the outer disk structure is surprisingly not in Keplerian rotation (Piétu et al. 2005; Lin et al. 2006). Since the disk is not self-gravitating, one plausible explanation would be the youth of the system.

Piétu et al. (2005) also find that both the mm dust and $\mathrm{CO}$ gas have a ring-like distribution with inner radius around 70-100 AU. The origin of the hole may be due to a substellar or very low-mass stellar companion located at 20-40 AU from the primary, as suggested by the recent VLA observations from Rodríguez et al. (2007) at $3.6 \mathrm{~cm}$.

Such a hole is not observed in the NIR where the dust opacity can be very high, hiding cavities with moderate contrasts (20-100), which are detectable in the $\mathrm{mm} / \mathrm{submm}$ range 

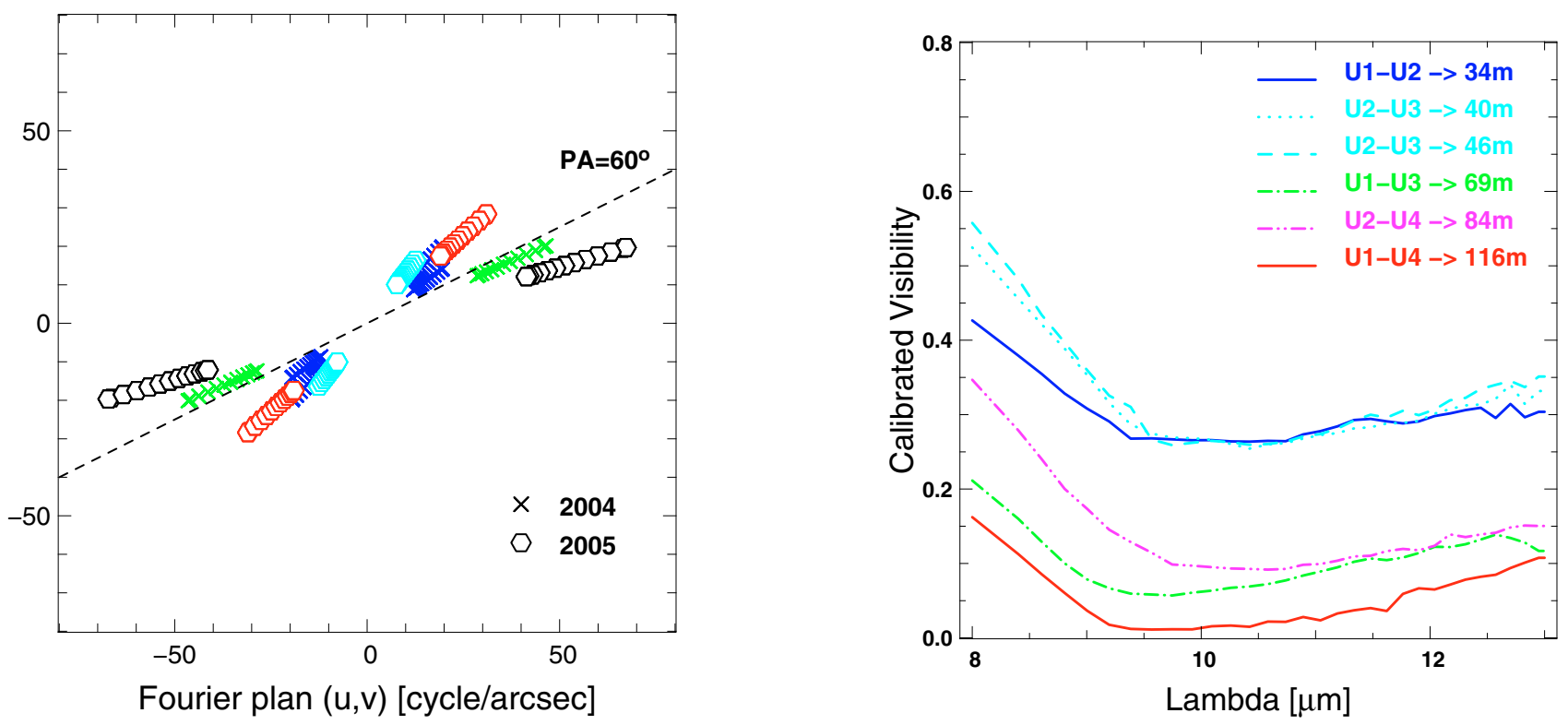

Fig. 1. Left: spatial frequency sampling with the five UT baselines of the VLTI. Our observations probe the stellar environment in the direction of the major axis of the outer disk (dashed line, see text). The radial extension of the $(u, v)$ tracks is linked to MIDI spectral dispersion. Right: low-resolution visibilities across the $\mathrm{N}$-band for the five UT baselines. The projected baseline length is indicated in the legend. The steep drop at short wavelengths is characteristic of the contribution of the unresolved, very inner disk. (See the electronic file for a color version)

(Piétu et al. 2006). A dust depletion has been recently reported by Oppenheimer et al. (2008) from NIR polarimetric observations at a radius of $102 \mathrm{AU}$ and $\mathrm{PA}=333 \mathrm{deg}$, although its link with the hole observed at mm-wavelength has still to be demonstrated. If AB Aur is a binary star, this also does not preclude optically thick gas and dust located in one or two individual disk(s) inside the Roche lobe of the system (Artymowicz et al. 1991). Indeed, hot dust very close to the star has been reported by NIR interferometers. Monnier \& Millan-Gabet (2002) and Monnier et al. (2005) have shown that, in such objects, the distribution of the circumstellar dust is truncated at an inner radius corresponding to the dust sublimation radius (which appears at temperatures of $\sim 1000-1700 \mathrm{~K}$, depending on the dust composition). Eisner et al. (2007) derive an inner rim location of 0.2-0.3 AU for $\mathrm{AB}$ Aur and suggest that the emission of hot gas might be taken into account to reproduce their PTI spectrally dispersed measurements in the $K$-band. Using the NIRSPEC echelle spectrograph on the Keck telescope, Brittain et al. (2007) observed $\mathrm{Br} \gamma$ and rovibrational $\mathrm{CO}$ emissions, which are due to a likely optically thick gaseous inner component. The $H$-band observations from IOTA by Millan-Gabet et al. (2006) also reveal nonzero closure phase measurements, which are interpreted as either a gravitational instability in the very inner disk linked to viscous heating or the existence of a stellar/substellar companion close to star (1-4 AU).

Both the large and small scales around AB Aurigae appear to have unusual morphologies and kinematics, far from the expected "proto-type" that is commonly modelled in the literature. However, the same magnitude for the inclination is found for the very inner disk observed by NIR interferometry techniques and the outer disk imaged from the NIR to the mm domain, with values ranging within $20-35^{\circ}$.

Results of a survey of Herbig Ae stars by Leinert et al. (2004) have clearly shown that MIDI, the interferometer of the VLT working in the range $8-13 \mu \mathrm{m}$, is very well-suited to studying the inner dust disks $(\sim 1-10 \mathrm{AU})$ of Herbig Ae/Be stars, in contrast to NIR interferometry, which probes the inner rim properties better. We thus observed AB Aurigae with MIDI (in the context of the Guaranteed Time Observations) in order to characterise its inner disk structure (scale-height, flaring ...) and dust properties. The observations and the data reduction are described in Sect. 2. Section 3 shows the observational results in terms of size and geometry of the emission. We present a global modelling of the dust disk in Sect. 4 and discuss the results in Sect. 5.

\section{Observations and data reduction}

\subsection{MIDI observations}

The observations reported in this paper were collected during two separate runs in December-January 2004/05 and 2005/06 with pairs of $8 \mathrm{~m}$ telescopes at the VLT interferometer (Chile). The beams were combined with the MIDI instrument (Leinert et al. 2004) operating in the $8-13 \mu \mathrm{m}$ range. Sparse measurements were obtained between 34 and $116 \mathrm{~m}$ of projected baseline length with the following interferometer configurations: U1-U2, $\mathrm{U} 2-\mathrm{U} 3, \mathrm{U} 1-\mathrm{U} 3, \mathrm{U} 2-\mathrm{U} 4$, and U1-U4. Figure 1 displays the $(u, v)$ plane of spatial frequencies covered by our observations. Given the high declination of the source $(\delta=+28 \mathrm{deg})$ for the Paranal site, the Earth rotation does not produce much synthesis effect and a very limited range of azimuth angles was covered. It should be noticed that most of them are globally co-aligned with the major-axis of the cold disk, whose direciton is represented by the dashed line at the position angle (PA $\simeq 60 \mathrm{deg}$ ) of the major axis determined by the $\mathrm{CO}$ millimetric maps of Piétu et al. (2005).

The transfer function of the instrument is monitored thanks to interleaved observations of bright standard (unresolved) stars, observed at a similar airmass. Among our measurements, six were obtained with the low dispersion mode (PRISM, $R=$ $\lambda / \delta \lambda=30)$ and one with the medium dispersion facility (GRISM, $R=\lambda / \delta \lambda=230$ ). For this, only the photometry could be usefully extracted, since it was not possible to make an absolute calibration of the visibility due to the lack of relevant interferometric calibrator star. 


\subsection{Interferometric data reduction}

The interferometric observable, namely the raw visibility or contrast, is computed from the integration of the power spectral density (PSD) of the interferometric signal around the spatial frequency determined by the ratio of the baseline and observing wavelength. This process follows three major steps: the evaluation of the photometric level, the computation of the PSD, and its integration in the relevant frequency domain, including subtracting the background noise.

Before combining the two telescope beams to produce the interferometric signal, the spectrum of the target is recorded to determine the photometric level in each beam, at the output of the optical train. The sky background, dominant in the thermal infrared, is subtracted thanks to a classical chopping procedure with a typical amplitude of $10^{\prime \prime}$ and a temporal frequency of $2 \mathrm{~Hz}$. The slit of the spectrograph spreads the target's flux over a variable number of pixels, dependent on the seeing conditions. All our measurements were recorded with the MACAO adaptive optics systems, that can partially compensate for the wavefront corrugations. The photometric signal of each telescope beam (recorded separately) is extracted with help of a numerical mask of Gaussian shape, that allows determining at each wavelength the position and extension of the illuminated pixels by fitting a Gaussian function of variable width in the direction perpendicular to the spectral dispersion.

The same mask is used to extract the two phase-opposite interferometric signals, that are subtracted to each other in order to minimize the contribution of correlated noise. The PSD of the resulting dispersed interferogram is integrated over the fringe peak and subtracted from the residual photon noise and background, whose level is determined on the basis of the lowest values in the PSD histogram. The raw visibility is finally obtained by dividing this quantity by the photometric flux, with a possible binning. We have used the MIA package ${ }^{1}$ to proceed to these various steps. We have modified the public version of the code to evaluate the internal uncertainties on the basis of the correlated flux distribution in each spectral channel and of the photometric fluctuations, whose uncertainties are propagated to derive the final error bars. The number of photometric frames recorded after the fringe tracking in the HIGH-SENS mode ranges from 1500 (first run) to 5000 (second run), thus yielding smaller and more robust uncertainties for the second data set.

\subsection{Determination of total uncertainties}

The total uncertainties presented include the contribution by the intrinsic dispersion of the correlated flux, of the photometric flux used to normalise the visibility, and by the transfer function itself (the last is almost negligible at very low values of correlated flux). The temporal fluctuations of the transfer function were generally observed to be larger in the $8-10 \mu \mathrm{m}$, especially during the 2004 run, when only 1500 photometric frames per beam were recorded after the fringes. Errors increase naturally at the edges of the filter. Typical uncertainties range from $5 \times 10^{-2}$ at $10 \mu \mathrm{m}$ to $10-15 \times 10^{-2}$ at $8 \mu \mathrm{m}$. At long baselines, and because of the low value of $\mathrm{AB}$ Aur visibilities, the transfer function only has a very weak influence on the visibility normalization, especially at long wavelengths $(10-13 \mu \mathrm{m})$, where the instrumental contrast is stabler.

\footnotetext{
1 http://WwW.mpia-hd.mpg.de/MIDISOFT/, http://www.strw. leidenuniv.nl/ nevec/MIDI/
}

Table 1. Log of observations.

\begin{tabular}{lccccc}
\hline \hline Date & Baseline & Bproj & BAzim & Mode & Reference star(s) \\
\hline $2004-12-27$ & UT2-UT3 & $40 \mathrm{~m}$ & $54 \mathrm{deg}$ & PRISM HD25604, HD37160 \\
$2004-12-27$ & UT2-UT3 & $46 \mathrm{~m}$ & $44 \mathrm{deg}$ & PRISM HD25604, HD37160 \\
$2005-01-01$ & UT2-UT4 & $84 \mathrm{~m}$ & $67 \mathrm{deg}$ & PRISM & HD37160 \\
$2005-12-22$ & UT1-UT2 & $34 \mathrm{~m}$ & $38 \mathrm{deg}$ & PRISM HD20644, HD39400 \\
$2005-12-23$ & UT1-UT3 & $69 \mathrm{~m}$ & $48 \mathrm{deg}$ & PRISM HD20644, HD34334 \\
$2005-12-25$ & UT1-UT4 & $116 \mathrm{~m}$ & $74 \mathrm{deg}$ & PRISM & HD20644 \\
\hline
\end{tabular}

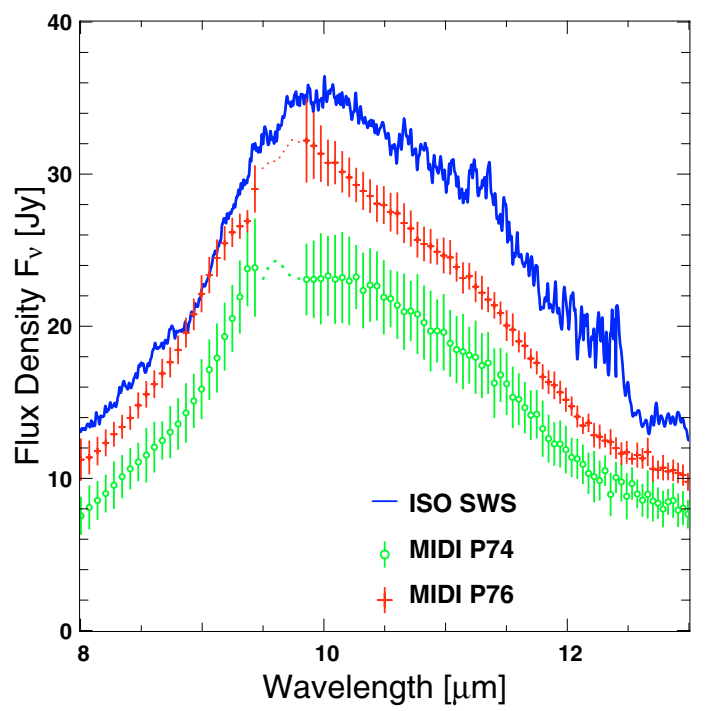

Fig. 2. Comparison between ISO and MIDI spectra (the FOV is $14^{\prime \prime} \times$ $20^{\prime \prime}$ and about 300 mas respectively). The flux measured by ISO SWS (thick, solid line) is about 1.35 times larger than the $\mathrm{N}$-band flux derived with MIDI in 2004 and at most 1.2 times larger in 2005. The atmospheric ozone absorption feature degrades the spectrum quality around $9.5 \mu \mathrm{m}$ (dots). The discrepancy between the two epochs might be representative of the already reported variability of the source at IR wavelength.

\section{Observational results}

\subsection{The $N$-band spectrum}

The spectrum measured by MIDI is presented in Fig. 2 for the two epochs separated by one year (denoted P74 and P76). The ISO spectrum displayed in Fig. 2 for the sake of comparison was obtained after reprocessing of the raw data with the software developed by (Sloan et al. 2003) ${ }^{2}$ in order to properly correct the mismatch between the different parts of the covered wavelength range between 11 and $13 \mu \mathrm{m}$. Absolute calibration of MIDI data makes use of reference stars observed at close airmass (see Table 1). The shape of the spectrum in the $N$-band is very smooth and dominated by the broad silicate feature in emission, already shown by ISO with the short-wavelength spectrograph (SWS), although this later includes a possible contribution from a large-scale circumstellar component due to its very broad field-of-view.

Although both MIDI and ISO spectra show a similar shape, the absolute flux measured with the UTs appears slightly weaker. Given a typical uncertainty in the ISO SWS absolute flux calibration of about $10 \%$ at these wavelengths, the discrepancy amounts to at most $35 \%$ of the ISO peak flux for the first epoch (P74) and varies from 0 to $20 \%$ for the second one (P76). Two

\footnotetext{
2 http://isc. astro. cornell. edu/ sloan/library/ swsatlas/atlas.html
} 

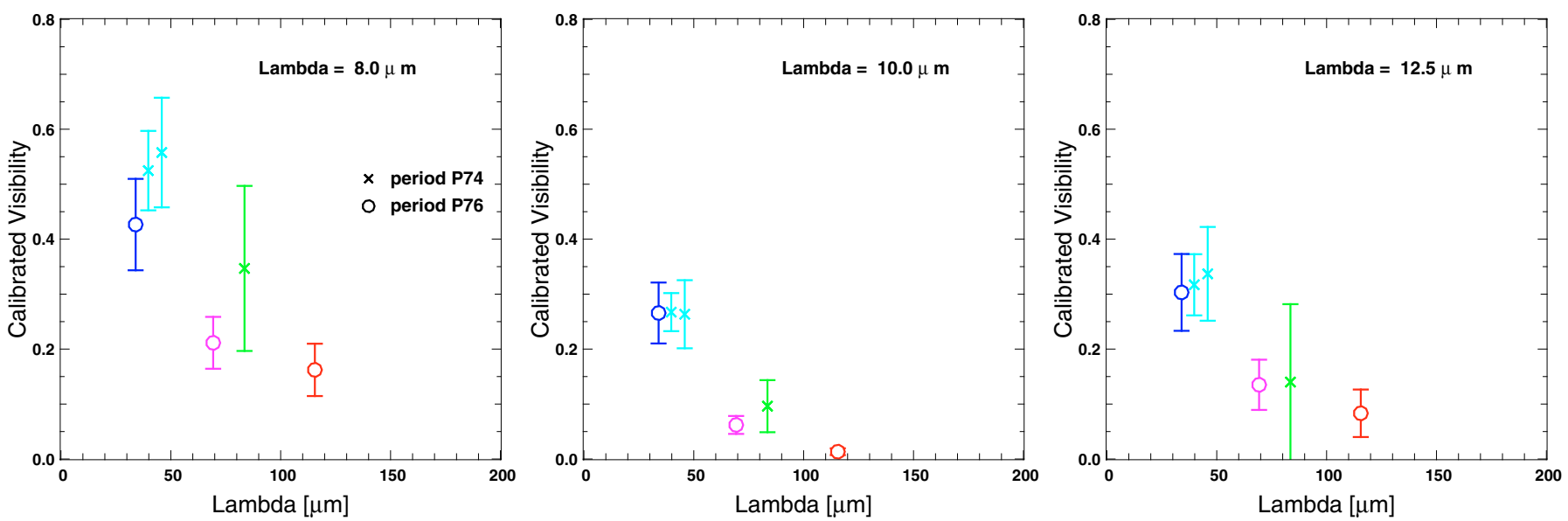

Fig. 3. Evolution of the visibility as a function of the projected baseline length for the three selected wavelengths, which are supposedly representative of the continuum $(8,12.5 \mu \mathrm{m})$ and $\mathrm{Si}$ line $(10 \mu \mathrm{m})$ emissions. The type and color of markers are associated with the observing epoch and baseline (see legend in Fig. 1).

possible origins for this discrepancy are the variability of the source at mid-IR wavelength and the possible contribution of a large-scale component, already detected through the scattered emission of small grains in the optical and near-IR domains (Grady et al. 1999; Fukagawa et al. 2004), which extends out to about $1000 \mathrm{AU}$. The broader width of the ISO beam could explain the increased discrepancy at longer wavelength, as it might be linked to a more extended component outside the MIDI field of view. The difficult absolute calibration of MIDI spectra is mainly linked to the variability of the water vapour content in the atmosphere during the night and to uncorrected airmass effects. The calibration of the P76 data is much more robust, thanks to the closer angular separation between the source and the reference stars and the level of flux detected is repeatable over the 3 nights. These second epoch spectra are almost consistent within the error bars with the ISO values. The temporal variability of the source at IR wavelength is proposed as the main origin for the observed discrepancy between the two epochs but should be confirmed by future observations. The 10 and $20 \mu \mathrm{m}$ fluxes reported in the literature present a variability of about $24 \%$ and $46 \%$, respectively (Chen \& Jura 2003), which has not been understood yet.

\subsection{Interferometric measurements and size of the emission}

The observed visibilities in the low resolution mode are reported in Figs. 1 and 3. Figure 1 displays the spectral dependence of the visibility across the $N$-band for the six measurements ( 2 short and 3 long baselines). The global behaviour can be characterised by a steep decrease of a factor 2 to 3 between 8 and $9.5 \mu \mathrm{m}$, where the visibility reaches its minimum. It is then followed by a plateau between 9.5 and $11 \mu \mathrm{m}$ and slowly/gradually rises up to $13 \mu \mathrm{m}$. Although this positive slope would not look significant given our absolute precision, it is systematically observed for each independent measurement and is thus believed to be real. The three short-baseline measurements $\left(B_{p}=34,40\right.$ and $\left.46 \mathrm{~m}\right)$ provide consistent visibilities within their uncertainty. At $8 \mu \mathrm{m}$, large uncertainties can be caused by temporal instabilities of the transfer function or of the photometry. The $34 \mathrm{~m}$ measurement has uncertainties dominated by the transfer function fluctuations, while the $69 \mathrm{~m}$ and $116 \mathrm{~m}$ observations are almost unaffected by this quantity. The long error bars of the $84 \mathrm{~m}$ measurement are due to its internal uncertainty (statistical dispersion of the raw visibilities and large skewness of their distribution).

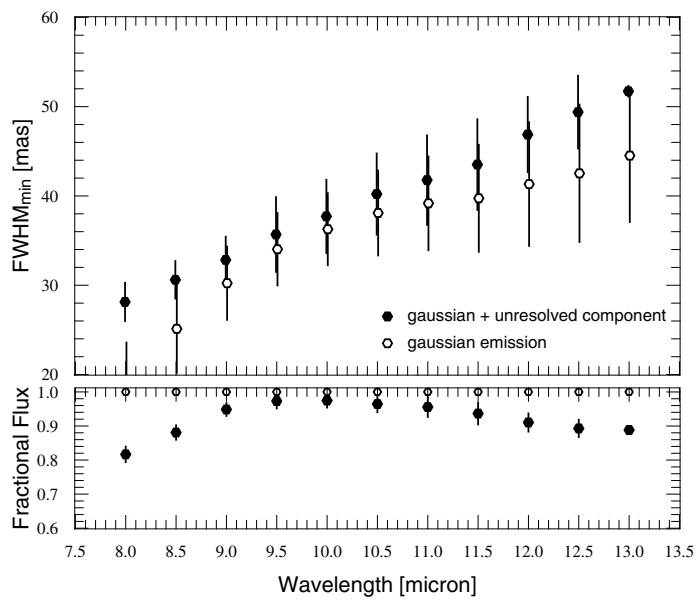

Fig. 4. Equivalent $F W H M$ size and fractional flux ratio for simple geometrical models composed of an unresolved component (very inner disk) surrounded by an extended Gaussian emission (full symbols) or a pure Gaussian emission (open markers). The FWHM is actually a lower limit to the emission size, since the environment is already resolved at the shorter baseline. The lower panel shows the contribution of the extended emission to the total flux for both models. The fit is based on the second-epoch measurements (December 2005).

Figure 3 displays amplitude visibilities as a function of the projected baseline for three selected wavelengths $(8,10$, and $12.5 \mu \mathrm{m})$. The 8 and $12.5 \mu \mathrm{m}$ channels are supposed to trace the continuum emission, while the $10 \mu \mathrm{m}$ channel stands for the centre of the silicate feature. Given the sparse spatial sampling and our uncertainties, the visibility shows a steady decrease from small to long baselines at all wavelengths, as expected for a resolved extended emission. In order to convert the visibilities into typical structure sizes, it is necessary to take the change in spatial resolution from 8 to $13 \mu \mathrm{m}$ into account. At the distance of the Taurus-Auriga star-forming region, a $100 \mathrm{~m}$ baseline provides a formal linear spatial resolution from 3 to $5 \mathrm{AU}$. We first computed sizes for a Gaussian model, although such a geometrical model has no physical sense and yields poor fits. Another simple model was considered, consisting of an unresolved component (to account for the very inner disk and rim, i.e. inside 1AU) surrounded by a Gaussian-shaped emission with adjustable size and flux ratio (see Fig. 4). It is believed to provide a more realistic picture of the stellar environment in the $8-10 \mu \mathrm{m}$ range and 
Table 2. Derived characteristics of the resolved emission for simple geometric models.

\begin{tabular}{rccc}
\hline \hline Wavelength & $8 \mu \mathrm{m}$ & $10 \mu \mathrm{m}$ & $12.5 \mu \mathrm{m}$ \\
\hline$F W H M^{a}$ [mas] & $28 \pm 2$ & $38 \pm 4$ & $49 \pm 4$ \\
[AU] & $4.6 \pm 0.3$ & $5.5 \pm 0.6$ & $7.1 \pm 0.6$ \\
$F_{\text {gauss }} / F_{\text {tot }}$ & $0.82 \pm 0.02$ & $0.97 \pm 0.02$ & $0.89 \pm 0.02$ \\
\hline$F W H M^{b}$ [mas] & $19 \pm 5$ & $36 \pm 4$ & $43 \pm 8$ \\
[AU] & $2.7 \pm 0.6$ & $5.2 \pm 0.6$ & $6.2 \pm 1.2$ \\
\hline
\end{tabular}

${ }^{a}$ Gaussian and unresolved emissions (lower limit of $F W H M$ size).

${ }^{b}$ Pure Gaussian emission (lower limit of FWHM size).

shows in particular the decreasing contribution of the unresolved rim when the wavelength increases.

The corresponding equivalent diameters, FWHM, and flux ratios are reported in Table 2 for the three selected wavelengths. These values actually provide lower limits for the size of the emitting region since the visibility is not constrained at baselines shorter than $34 \mathrm{~m}$. Typical FWHM sizes range between 20 and 50 mas from 8 to $13 \mu \mathrm{m}$ (or 3 to $8 \mathrm{AU}$ of projected distance). At $11.7 \mu \mathrm{m}$, the derived minimum $F W H M$ size of $7 \mathrm{AU}$ is about half that measured by Mariñas et al. (2006) with Gemini North at the same wavelength, while Chen \& Jura (2003) have not resolved the environment with Keck and provide an upper limit of $0.3^{\prime \prime}$ (43 AU). At $10.6 \mu \mathrm{m}$, Liu et al. (2005) report an FWHM size of 24-30 AU with nulling interferometric techniques at the MMT (although with a rather large inclination that disagrees with other recent studies). These larger mid-IR sizes are still compatible with our measurement, so the differences may indicate that the brightness radial profile cannot be safely modelled by a simple Gaussian.

\subsection{Disk geometry}

Since the Fourier plane was mostly sampled around one main azimuth angle, as illustrated in Fig. 1, it is not possible to properly constrain the inclination of the inner disk from our observations. Nevertheless, this azimuth direction is aligned with the PA of the major axis $(\simeq 60 \mathrm{deg})$ of the outer disk revealed by the recent CO observations of Piétu et al. (2005) and by the near-IR scattered light images of Fukagawa et al. (2004). It is also consistent with the value derived by $\mathrm{H} \alpha$ polarimetric measurements, PA 70 deg (Pontefract et al. 2000). Most of the various mid-IR, large-aperture observations reported in the literature agree with a disk seen almost face-on. The near-IR interferometric measurements of the very inner disk are also consistent with a small inclination angle (Eisner et al. 2004). We therefore assume in the following that the inner disk probed with MIDI has the same inclination and orientation as the outer disk and that our interferometric measurements trace the major axis of this disk (thus neglecting the small azimuth variations).

\section{Global modelling}

In this section, we present the result of the modelling performed with the radiative transfer code MC3D developed by Wolf et al. (1999) and Wolf (2003). MC3D is based on the Monte-Carlo method, designed to solve the radiative transfer problem selfconsistently and can produce multiple wavelength images and spectral energy distributions of accretion disks. The possible heating sources are provided by 1) the central star and 2) the accretion. The accretion luminosity was not taken into account in the following. To reproduce the basic photometry and spatial structure of the AB Aur disk at mid-IR wavelengths, we investigated a simple and classical distribution of dust grains in a flared, passive disk. In this approach, we neglect the influence of any large-scale envelope and focus on the characteristics of the dust disk. In the following sections, we describe our model, our choice for its inputs parameters, as well as our minimisation method. In Sect. 4.4, we present the most probable model, emphasising the specific contribution of the mid-IR interferometric observations with respect to spectro-photometry-based analyses.

\subsection{A simple flared disk}

Our model consists of a passive flared disk, for which we assume a volume density profile described by the following equation, successfully used to model the outer regions of the circumstellar disk around HH 30 IRS (Wood et al. 2002) or that of the Butterfly Nebula (Wolf et al. 2003):

$\rho(r, z)=\rho_{0}\left(\frac{r}{r_{0}}\right)^{-\alpha} \exp \left\{-\frac{1}{2}\left[\frac{z}{h(r)}\right]^{2}\right\}$

where $r$ is the radial distance to the star in the disk midplane, $z$ the altitude above the midplane, $r_{0}$ a reference distance and $h(r)$ the scale height, which follows the equation

$h(r)=h_{0}\left(\frac{r}{r_{0}}\right)^{\beta}$.

The coefficients $\alpha$ and $\beta$ respectively describe the radial dependence of the volume density and the flaring of the disk. The scale height is scaled at the radial distance of $r_{0}=100 \mathrm{AU}$ (and will be written as $h_{0}=h_{100}$ in the following). At the inner radius, the geometry is that of a vertical wall, without puffed-up rim, because we focus on the mid-IR observables.

In early theoretical works, disk models are vertically isothermal with a slowly rising surface $h / r \propto r^{1 / 8}$ (i.e., $\beta=9 / 8=$ 1.125) corresponding to $T \propto r^{-3 / 4}$ (Kenyon \& Hartmann 1987). Relaxing the assumption of isothermal structure, Chiang \& Goldreich (1997) have developed a 2-layer model with a superheated photosphere, which leads to $h / r \propto r^{2 / 7}(\beta=9 / 7 \approx 1.29)$ in the first $\sim 100$ AU. Models by D'Alessio et al. (1998, 1999) considering the calculation of the vertical structure of disks surrounding T Tauri stars results in temperature gradients that yield $h / r \propto r^{1 / 4}(\beta=5 / 4=1.25)$ beyond a few AUs.

In our case, we assume that the coefficients $\alpha$ and $\beta$ are linked through the relation $\alpha=3(\beta-1 / 2)$. This equation assumes that the power index of the surface density law $p$ and of the temperature $q$ are linked by $p+q=3 / 2$.

Grain properties. Since the system is relatively young (1$2 \mathrm{Myr}$ ) and the dust appears less evolved than in other systems (Piétu et al. 2005), we follow the classical MRN dstribution law (Mathis et al. 1977):

$n(a) \propto a^{-3.5}, \quad$ with $\quad a_{\min }<a<a_{\max }$

where $a$ is the grain size, $a_{\min }=5 \mathrm{~nm}$ and $a_{\max }=250 \mathrm{~nm}$ (interstellar medium distribution). We did not take possible grain growth or vertical sedimentation into account in the present work.

\subsection{Choice of the parameters}

Since AB Aur is one of the most observed Herbig stars, we have taken advantage of the previous multi-wavelengths studies to 
Table 3. Adopted and fitted parameters for our model of AB Aur disk.

\begin{tabular}{lcc}
\hline \hline Stellar fixed parameters & & Ref. \\
\hline Stellar Luminosity $\left(L_{*}\right)$ & $47 L_{\odot}$ & 8 \\
Effective Temperature $\left(T_{\text {eff }}\right)$ & $9333 \mathrm{~K}$ & 8 \\
\hline Adopted fixed parameters & $144 \mathrm{pc}$ & Ref. \\
\hline Distance $D$ & $0.30 \mathrm{AU}$ & 5,6 \\
Inner Radius $\left(R_{\text {in }}\right)$ & $200 \mathrm{AU}$ & 3 \\
Outer Radius $\left(R_{\text {out }}\right)$ & $33 \mathrm{deg}$ & $2,3,6,7$ \\
Inclination $(i)$ & $5 \times 10^{-5} M_{\odot}$ & $1,2,3,4$ \\
Dust Mass $\left(M_{\mathrm{d}}\right)$ & $2 \mathrm{~g} \mathrm{~cm}{ }^{-3}$ & \\
Dust grain Density & $62.5 \%$ amorp. silicates & \\
Dust Composition & $37.5 \%$ graphite & \\
Grains size & {$[0.005-0.250] \mu \mathrm{m}$} & \\
Sublimation Temperature $\left(T_{\text {sub }}\right)$ & $1500 \mathrm{~K}$ & \\
& & \\
\hline Fitted parameters & Value $[ \pm 3 \sigma]$ & $\chi_{\mathrm{r} \text { tot }}^{2}$ \\
\hline$\quad$ With ISO spectrum & $1.27[ \pm 0.025]$ & 2.93 \\
$\beta \quad$ & $8.0[ \pm 0.5]$ & \\
$h_{100}$ [AU] & & \\
$\beta \quad$ With MIDI spectrum & $1.27[ \pm 0.025]$ & 3.74 \\
$h_{100}$ [AU] & $8.0[ \pm 0.5]$ & \\
\hline
\end{tabular}

Ref.: 1) Mannings \& Sargent (1997); 2) Semenov et al. (2005); 3) Piétu et al. (2005); 4) Corder et al. (2005); 5) Millan-Gabet et al. (2001); 6) Eisner et al. (2004); 7) Pantin et al. (2005); 8) Hernández et al. (2004).

restrict the free-parameter space. The geometrical parameters, such as the outer and inner radii and the inclination, were fixed according to the literature. The model parameters are given in Table 3 .

Size and inclination. Following Eisner et al. (2007), we have first adopted $R_{\text {in }}=0.3 \mathrm{AU}$ for the inner radius of the dust disk. This value is also representative of the typical inner radii derived by Monnier et al. (2005) for Herbig stars with $50 L_{\odot}$. The determination of the dust destruction radius is, however, a complex process, as discussed in Isella \& Natta (2005) and Tannirkulam et al. (2007). The location and shape of the rim can strongly depend on the nature, size, and absorption properties of the considered grains, on the gas pressure within the rim, or on the vertical sedimentation of the grains, as well as on the possible presence of an optically thin or thick gas component between the stellar photosphere and the inner dust wall. For the sake of simplicity, we use a vertical wall at the inner rim position.

The outer disk radius $\left(R_{\text {out }}\right)$ is fixed to $200 \mathrm{AU}$, based on the millimetric and mid-IR observations (Piétu et al. 2005; Pantin et al. 2005; Mariñas et al. 2006). The faint mid-IR emission, which is detected at a greater distance, is too extended to affect the interferometric measurement, whose FOV is only $0.6^{\prime \prime}$ (or $70 \mathrm{AU})$ at $\lambda=10 \mu \mathrm{m}$. This input parameter is mainly used in the code to determine the radial structure of the density profile.

The inclination of the inner disk on the sky plane is set to $33 \mathrm{deg}$, a value consistent with an almost face-on configuration as derived on a large scale from scattered light images ( $i=30 \pm 5 \mathrm{deg}$, Fukagawa et al. 2004) and mm-maps (20-40 deg, Piétu et al. 2005), or at high angular resolution in the near-IR (0-20 deg, Eisner et al. 2004).
Dust composition. The strong $10 \mu \mathrm{m}$ feature observed in the spectrum of $\mathrm{AB}$ Aur indicates that silicates grains dominate in the hot dust surface layer. Accordingly, we started our analysis with a classical mixture of astronomical silicates and carbon. The mixture is composed of $62.5 \%$ astronomical silicates (Weingartner \& Draine 2001) and $37.5 \%$ graphite, with a relative abundance of 2:1 between the two components of the anisotropic crystal structure of graphite. Bouwman et al. (2000) have analysed the ISO data and used a comparable mixture consisting of about $70 \%$ of silicates to reproduce the ISO spectrum.

Density law and mass. The mass of the disk is linked to $\rho_{0}$ after Eq. (1). Estimating the total mass remains a difficult task for several reasons. The various optically thin radio observations have inferred a total (ie. gas+dust) disk mass in the range 1.5-2.5 $\times 10^{-2} M_{\odot}$ (Mannings \& Sargent 1997; Semenov et al. 2005; Piétu et al. 2005; Corder et al. 2005). However, these estimates are relevant mainly for the outer disk. Piétu et al. (2005) highlight a drop of the continuum and $\mathrm{CO}$ emissions in the central $100 \mathrm{AU}$. Contrary to mm observations, the MIDI data trace the optically thick inner disk. Adopting a widely assumed gasto-dust ratio of 100:1, we estimate the maximum dust mass of the modelled disk to be a few $10^{-4} M_{\odot}$ and we use a fixed mass of $5 \times 10^{-5} M_{\odot}$ for our reference model.

Temperature. The temperature is self-consistently calculated by MC3D. We have adopted a maximum temperature of $T_{\text {sub }}=$ $1500 \mathrm{~K}$ for the sublimation of the dust.

Running the model. The adopted input parameters are given in Table 3. For this set of fixed parameters, we have explored a grid of $\left(h_{100}, \beta\right)$ values with $h_{100}$ in the range [5; 15] AU and $\beta$ varying from 1.10 to 1.50 . Taking the best parameters for $\beta$ and $h_{100}$, we then investigate the influence of the following parameters: the dust mass, the inner radius, the silicate content, the grain size, and the inclination.

\section{3. $\chi^{2}$ minimisation}

On the basis of the radiative transfer results, multi-wavelength images (from which visibilities are derived in the FOV of the interferometer) and the spectral energy distribution of the simulated disk are computed. The visibilities are calculated for eleven values of lambda in the $8-13 \mu \mathrm{m}$ range. Since we are focussing on the mid-IR domain, the synthetic SED is compared to the ISO measurements between 8 and $50 \mu \mathrm{m}$, where the luminosity of the star and that of the hot rim are negligible in comparison to the thermal emission of the upper layer of the disk.

Three $\chi^{2}$ maps are built separately for the SED, for the visibility, and for the combined constraints according to the below equations:

$$
\begin{aligned}
& \chi_{\text {sed }}^{2}=\sum_{\lambda_{i}=1 \ldots N_{\text {sed }}}\left(\frac{F_{v}^{\text {model }}\left(\lambda_{i}\right)-F_{v}^{o b s}\left(\lambda_{i}\right)}{\sigma_{F_{v}}}\right)^{2} \\
& \chi_{\text {vis }}^{2}=\sum_{\lambda_{j}=1 \ldots N_{\text {vis }}} \sum_{k=1 \ldots 5}^{\text {baselines }}\left(\frac{V i s^{\text {model }}\left(\lambda_{j}, B_{k}\right)-V i s^{\operatorname{MIDI}}\left(\lambda_{j}, B_{k}\right)}{\sigma_{V i s}}\right)^{2} \\
& \chi_{\text {tot }}^{2}=\chi_{\text {sed }}^{2}+\chi_{\text {vis }}^{2} \\
& \chi_{\mathrm{r} \text { sed }}^{2}=\chi_{\text {sed }}^{2} /\left(N_{\text {sed }}-2\right) ; \quad \chi_{\mathrm{r} \text { vis }}^{2}=\chi_{\text {vis }}^{2} /\left(N_{\text {vis }}-2\right) \\
& \chi_{\mathrm{r} \text { tot }}^{2}=\chi_{\text {tot }}^{2} /\left(N_{\text {sed }}+N_{\text {vis }}-2\right) .
\end{aligned}
$$

For the spectral energy distribution, we successively consider the ISO spectrum in the $8-50 \mu \mathrm{m}$ range $\left(N_{\text {sed }}=30\right)$ and the 

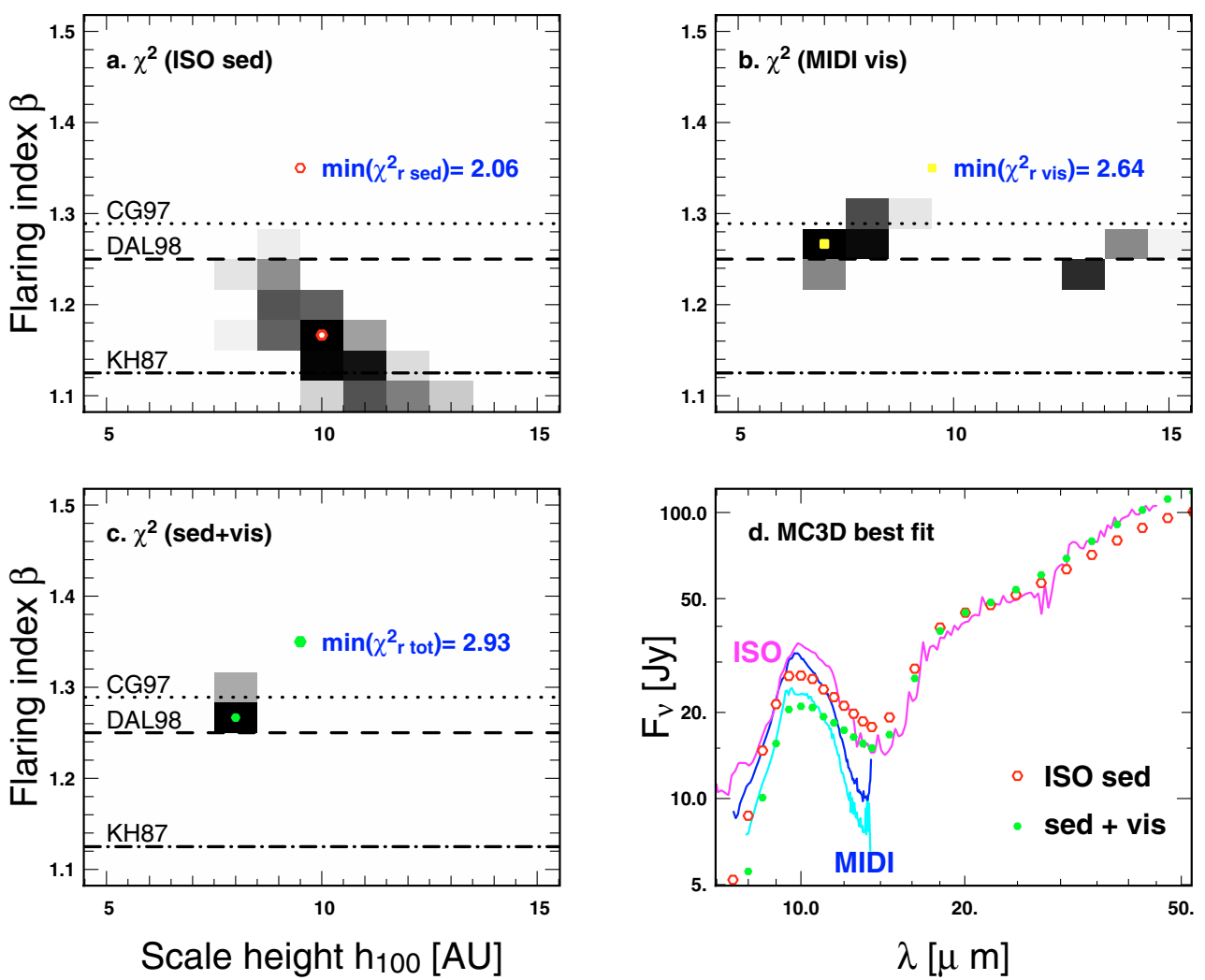

Fig. 5. Results of the disk modelling: $\chi^{2}$-maps for a flared disk model with a mass $M_{\text {dust }}=5 \times 10^{-5} M_{\odot}$, inner and outer radii $R_{\text {in }}=0.3 \mathrm{AU}$, $R_{\text {out }}=200 \mathrm{AU}$, an inclination $i=33 \mathrm{deg}$, with $62 \%$ of amorphous silicate grains following the MRN distribution. The flaring index $\beta$ and the scale height $h_{100}$ are the free parameters. a) SED fit alone based on ISO spectrum $(8-50 \mu \mathrm{m})$. b) $N$-band visibility fit alone based on MIDI observations. c) Combined fit. The grey scale ranges from the minimum of $\chi^{2}$ up to $6 \sigma$. The horizontal lines stand for the values of $\beta$ associated with the theoretical models of Kenyon \& Hartmann (1987) (dash-dot), D’Alessio et al. (1998) (dash), and Chiang \& Goldreich (1997) (dots). d) Best-fit synthetic SED compared to ISO (solid black line) and MIDI spectra (grey).

MIDI spectrum associated with the smaller FOV of the UTs $\left(N_{\mathrm{SED}}=11\right)$. The MIDI visibilities encompass 11 spectral channels over 5 baselines $\left(N_{\text {vis }}=55\right)$. With these numbers, the interferometric measurement has a slightly predominant weight in the $\chi^{2}$ minimisation process.

\subsection{Results}

As discussed in Sect. 3.1, the $N$-band spectrum measured with MIDI in the $\sim 300$ mas FOV of the UTs is very similar to the wide field-ISO spectrum, both in shape and in amplitude. Most of the emission detected with ISO seems to emanate from the central component, which we aim at modelling in this section. The ISO SED covers in addition a broader range of wavelengths, which is also helpful for characterising the disk emission and thus yields a valuable complementary constraint to determine its morphology. We therefore build our analysis on the basis of the combination of MIDI visibilities and the ISO spectrum first. The MIDI spectrum will serve in a second step to illustrate the influence of the aperture size on our conclusion.

\subsubsection{Combining MIDI visibilities with the ISO spectrum}

With the considered simple disk model, solutions can be found that closely reproduce both the spectral energy distribution and the visibility measurements in the $N$-band. The $\chi^{2}$ maps displayed in Fig. 5 illustrate the degeneracy of each observational constraint alone and demonstrate that both techniques can be advantageously combined to converge toward a unique set of parameters, thus alleviating the intrinsic degeneracy of each observable.

In Fig. 5, the SED fit is based on the ISO SWS spectrum with arbitrary total uncertainties of $15 \%$ (including the absolute calibration), which should be representative of the long-wavelength side of this spectrum and slightly conservative in the short wavelength range (e.g., see Kessler 2000). The first map (a) illustrates the coupling between the flaring index $(\beta)$ and the scale height $\left(h_{100}\right)$ as a result of their combined contribution to the total light reprocessed by the grains in the disk atmosphere, i.e. to the total integrated flux. A flatter disk requires a larger scale-height for the super-heated layer to directly capture and reprocess more stellar radiation and to produce a given observed luminosity. Conversely, the visibility fit (b) yields a unique value of $\beta$, but is degenerated for the scale height determination. This is due to the system inclination, as AB Aur disk is seen almost face-on (fixed $i=33 \mathrm{deg}$ ). The radial profile of the emission measured by the interferometer is fairly insensitive to the thickness of the disk along the line of sight in this configuration. With the given input parameters, the best-fit index $\beta \sim 1.27 \pm 0.025$ is intermediate between the predicted theoretical values 1.25 (D'Alessio et al. 1999) and 1.289 (Chiang \& Goldreich 1997) (horizontal lines). We show in Sect.5 that the inferred precision is actually dominated by uncertainties on the disk input parameters (e.g., dust mass). The combination of both constraints (c) provides a 

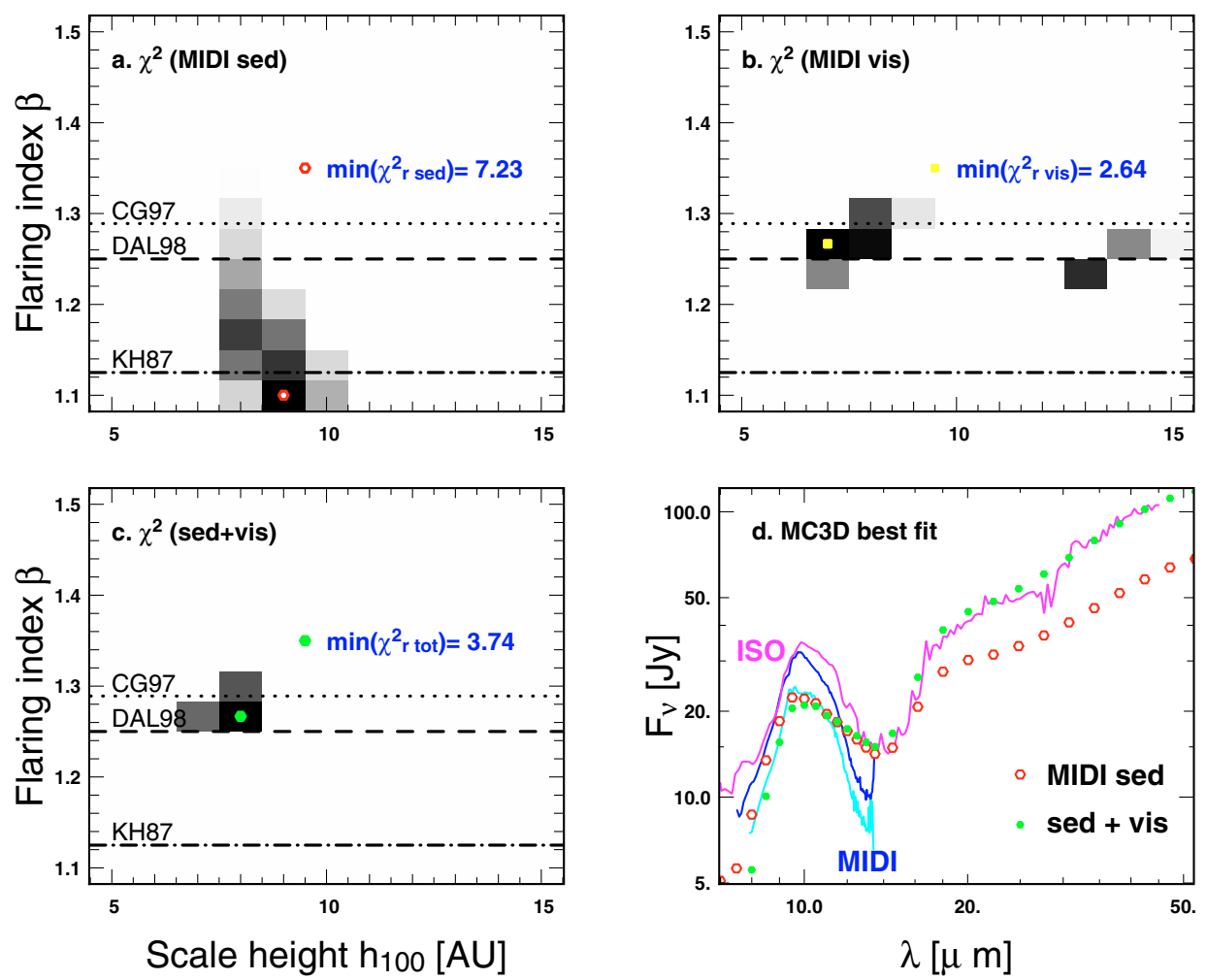

Fig. 6. Same as Fig. 5 above, but the ISO values are replaced with the MIDI spectrum in the fit. (See the electronic file for a color version).

unique intersection of the $\chi^{2}$ maps, hence a unique set of parameters for the disk morphology. The best solution for the combined fits yields: $\beta=1.27 \pm 0.025, h_{100}=8 \pm 0.5 \mathrm{AU}$, where the $1 \sigma$ statistical uncertainties are given by $\chi^{2}-\chi_{\min }^{2}=2.3$ and are derived from a higher resolution $\chi^{2}$-map. Figure 8 illustrates the variation in the model scale-height with the radial distance in the inner disk that is probed by our interferometric measurement, i.e., within the first $10 \mathrm{AU}$. We emphasise that these values are only valid in the framework of this model, with the input parameters listed in Table 3 . The values and the derived errors also weakly depend on the definition of the $\chi^{2}$ (number and choice of wavelengths and respective weight between interferometry and spectro-photometry).

The comparison between the modelled and the observed spectra is shown for the best-fit models in panel (d), considering the SED fit and the total fit separately. Both models are very close to each other and both reproduce the dust continuum emission beyond $13 \mu \mathrm{m}$. In the silicate resonance band, the agreement is not as good (although still acceptable, since the flux is plotted on a log-log scale), the modelled spectrum tends to underpredict the emission at shorter wavelengths. Remarkably, the model flux values match the MIDI spectrum closely in this wavelength range. The comparison is also shown for the visibilities on the five baselines in Fig. 7. The calculation confirms that the discrepancy between the visibilities along the major axis (squares) and minor axis (triangles) of the disk is small for the adopted inclination of $33 \mathrm{deg}$, thus justifying our assumption of co-aligned baselines a posteriori. The level and the shape of the visibilities are reproduced by our simple model: in particular, the steep drop at short wavelength and the slightly rising plateau between 10 and $13 \mu \mathrm{m}$. The discrepancy between the various fits is negligible compared to the uncertainty of our interferometric measurement and is also justified by the greater weight attributed to this in the fitting process.

\subsubsection{Combining MIDI visibilities with the MIDI spectrum}

In a second step, we used the MIDI spectrum, together with the visibilities, to constrain the model (see Fig. 6). Since our model is too simple to reproduce the shape of the silicate band in detail, we decided to halve the absolute calibration precision (photometric accuracy) of the MIDI spectrum. This means that we arbitrarily give more weight to the visibilities, hence to the disk morphology. This fitting process is thus more sensitive to the integrated flux over the $N$-band than to the spectrum shape.

Refining the dust composition and the geometry of the disk rim (responsible for the emission of hot dust) may help for improving the SED fitting, particularly in the short wavelength range. It is nevertheless out of the scope of this paper and should be further investigated.

Remarkably, the combined fit (Fig. 6d) matches the ISO spectrum at long wavelengths with a very similar slope in the 14-50 $\mu \mathrm{m}$ domain. Most of the mid-IR emission thus appears convincingly modelled by a simple flared disk with a negligible contribution from other possible components (e.g. large-scale envelope).

\section{Discussion}

\subsection{The disk flaring and the radial structure}

In the standard disk description (see Sect. 4.1), the flaring of the $\operatorname{disk} \beta$ is the main parameter linked to the temperature gradient in the disk photosphere. The best-fit values obtained in our simulations are consistent with a disk that is more flared than the canonical model of Kenyon \& Hartmann (1987) $(\beta=9 / 8)$ and agree more with those derived by D'Alessio et al. (1999) $(\beta=5 / 4)$. The visibility constraint for our reference model favours values 

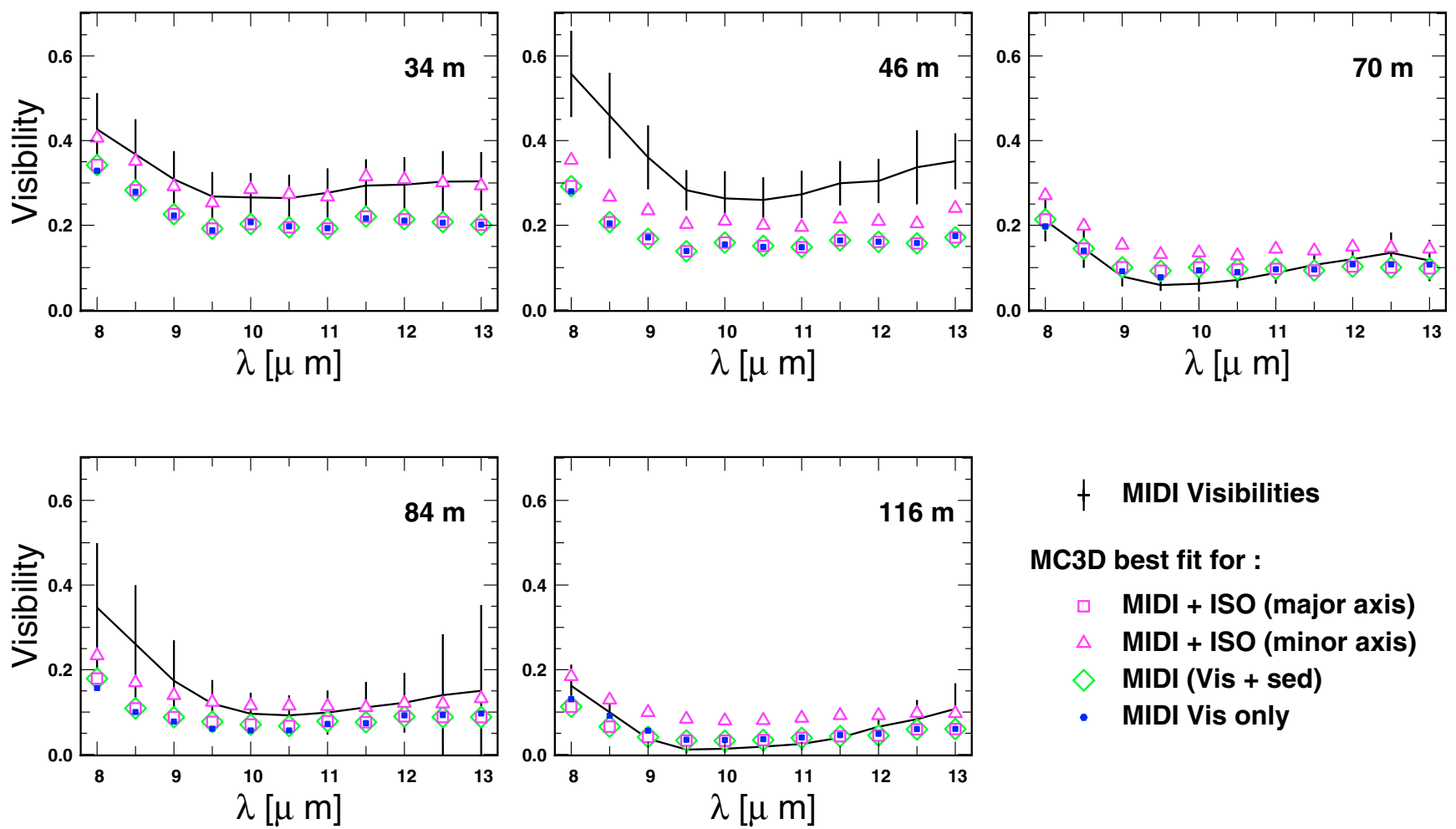

\section{† MIDI Visibilities \\ MC3D best fit for : \\ $\square \quad$ MIDI + ISO (major axis) \\ $\triangle \quad$ MIDI + ISO (minor axis) \\ $\diamond$ MIDI (Vis + sed) \\ - MIDI Vis only}

Fig. 7. MIDI observations compared to the best-fit models presented in Figs. 5 and 6. Squares (triangles) stand for the simulated visibilities along the major (resp. minor) axis of the modelled disk. VLTI observations are presumably aligned with the major axis. We present the best-fit models for the interferometric constraint alone (dots) and the combination of MIDI visibilities with the MIDI spectrum (diamonds) or the ISO spectrum (squares and triangles). The visibility constraint has a greater weight than the spectrum in the fitting process (see text).

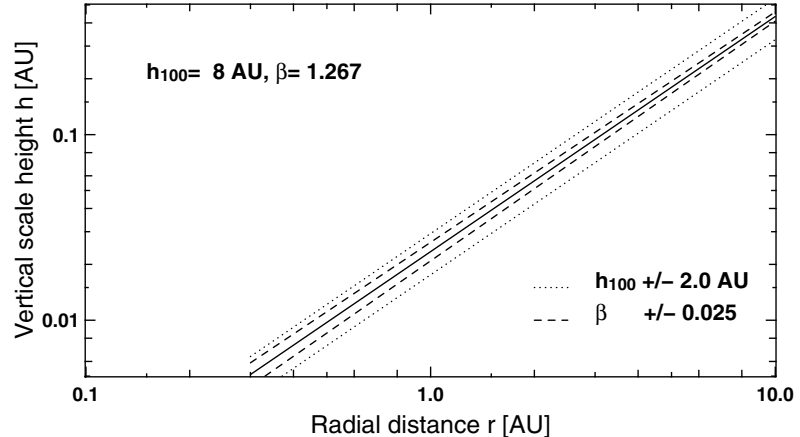

Fig. 8. Variation in the scale height in the very inner disk for the best-fit model presented in Fig. 5 and influence of a small change of $h_{100}$ and $\beta$. The inner rim is described by a simple vertical wall.

of $\beta$ in the range $1.25-1.30$, slightly higher than those inferred from the SED-fit alone. We show in the next section that this estimation is quite robust, even when small variations of the model input parameters are considered. Piétu et al. (2005) derived $\beta=1.4$ from the ${ }^{13} \mathrm{CO}$ maps, a value significantly higher in the outer regions of the disk (80-1300 AU). The associated scale height at $100 \mathrm{AU}$ is very close to our best-fit value with $h_{100}=8.5 \mathrm{AU}^{3}$. If assuming a Keplerian velocity field in the inner regions of the disk (not completely guaranteed in the case of $\mathrm{AB}$ Aur if we extrapolate to smaller radii the conclusions of Piétu et al. 2005), the radial gradient of temperature in the disk photosphere probed by our mid-IR observations is $T \propto r^{-q}$ with $q=3-2 \beta \simeq 0.5-0.52$ for $\beta \simeq 1.25-1.30$.

3 Their definition of the scale height differs from ours by a factor $\sqrt{2}$.
Similarly, we can compare our results for the radial surface density power index with that estimated by large-scale observations. The assumed $\alpha / \beta$ combination yields $\alpha \simeq 2.25-2.40$ and thus for the surface density $\left(\Sigma(r) \propto r^{-p}\right) p=\alpha-\beta \simeq 1.0-1.1$. This value is slightly lower than the one usually adopted for the young solar system $(p=1.5)$ and significantly lower than the index derived from mm observations for the outer annulus ( $p \simeq 2.3-2.7$ ). Discarding the previously adopted $\alpha=3(\beta-0.5)$ relation to fix $p=2.5$, we have checked that the fit would lead to a little increased flaring index $(\beta=1.3)$ and to a larger scale height $\left(h_{100} \sim 10.5 \mathrm{AU}\right)$, still in the same order as that derived from mm observations. It should, however, be emphasised that the above-mentioned mm interferometric observations have shown a dramatic change in the disk properties inside $100 \mathrm{AU}$, so that extrapolating these morphological parameters to the inner regions might be quite hazardous.

\subsection{Influence of input parameters}

In this section, we investigate the dependence of the best-fit values $\beta$ and $h_{100}$ on the main model input parameters to highlight possible hidden degeneracies in our analysis. Figures 9 to 11 present the best-fit values for the various models, each point being derived from a combined $\chi^{2}$-map, like those presented in Fig. 5c. The associated $\chi_{\text {redtot }}^{2}$ are close to each other and the associated models are almost equally probable.

1. The dust mass. $M_{\text {dust }}$ was varied from $5 \times 10^{-6}$ to $1 \times$ $10^{-4} M_{\odot}$. The upper value is in line with the typical masses used in the litterature to fit the mid-IR spectrum (e.g., Bouwman et al. 2000), while the lower value corresponds to our estimation of the dust amount, that would produce the 


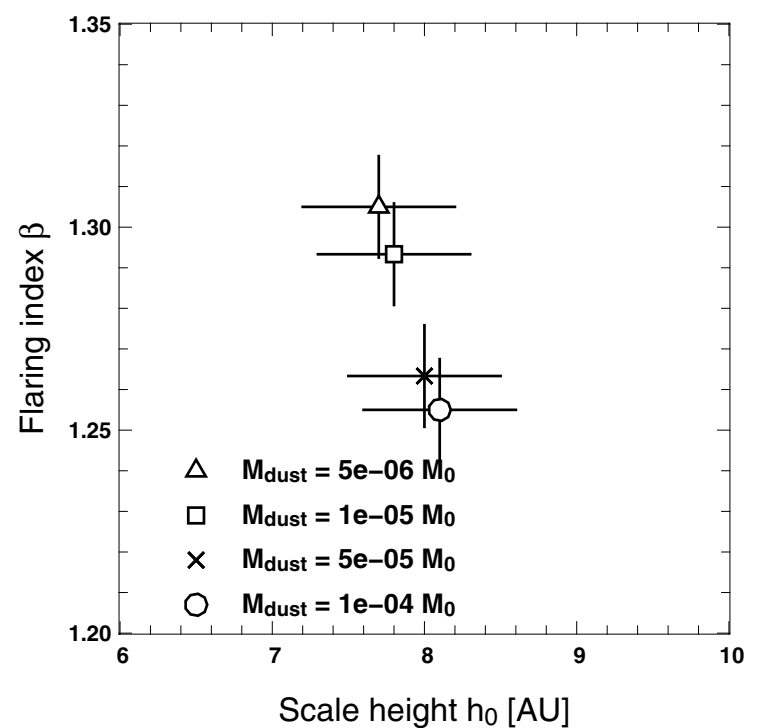

Fig. 9. Influence of $M_{\text {dust }}$ on the best-fit values of the flaring index and scale height in the disk (same diagram as in Fig. 5c). The input masses are symbolised with different markers. The other parameters are listed in Table 3.

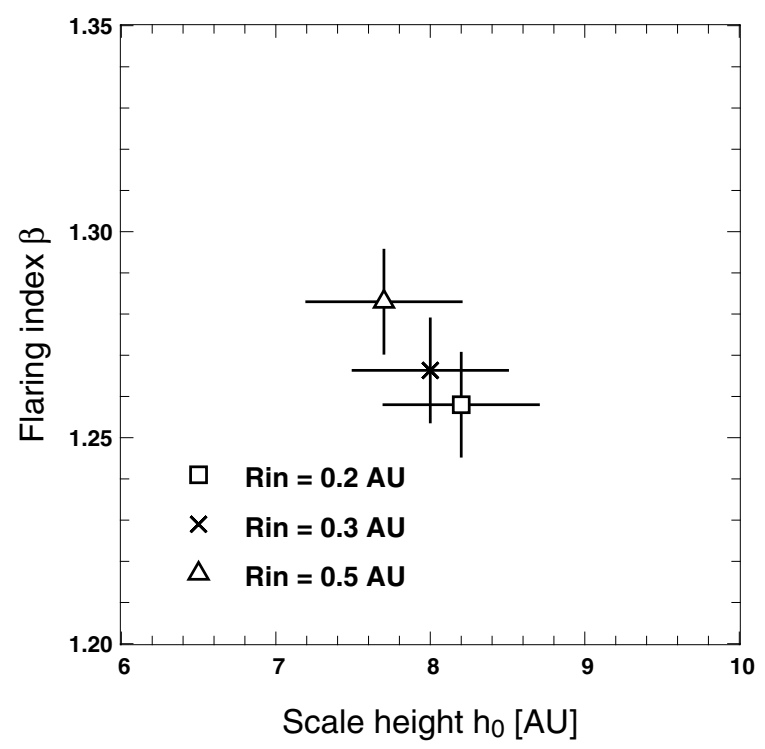

Fig. 10. Same diagram as in Fig. 9 for the disk inner radius $R_{\text {in }}$.

residual mm flux observed in the $\sim 70$ AU depleted zone evidenced by Piétu et al. (2005). Decreasing the dust mass does not modify the SED $\chi^{2}$ map significantly (although masses lower than $10^{-5} M_{\odot}$ yield less probable solutions), while the interferometric constraint clearly favours higher values of $\beta$. An increased dust mass corresponds to higher opacities and leads to flatter disks. This is associated with a weak increase in the scale height as $\beta-h_{100}$ variations are locked through the unchanged SED constraint. For the range of masses explored, the fitted $h_{100}$ remains around $8 \mathrm{AU}$, whereas $\beta$ can vary from 1.25 to 1.31 . This analysis does not provide a constraint on the true mass in the disk, since most of the dust mass is kept in the optically thick mid-plane region, which can only be probed at longer wavelengths.

2. The inner radius. Changing the position of the truncation radius does not affect the SED maps much since only the

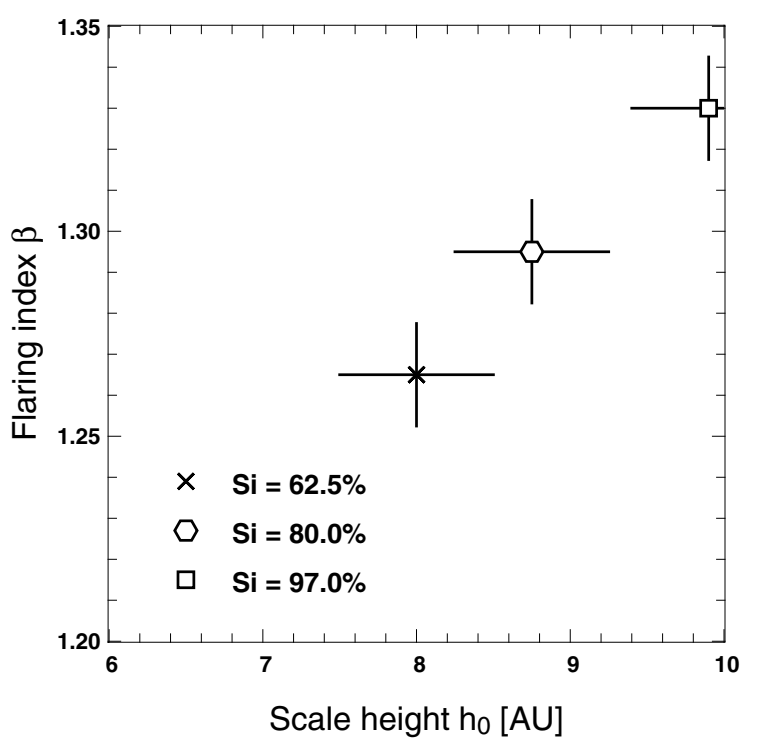

Fig. 11. Same diagram as in Fig. 9 for the abundance of amorphous silicates in the grain mixture.

short-wavelength domain can be influenced. The change in flaring index is nevertheless noticeable, $\beta$ increasing with $R_{\text {in }}$ in order to compensate for the lack of emission in the most inner part of the disk. The best-fit scale height drops subsequently because of the SED-degeneracy. The value of $R_{\text {in }}$ cannot be directly constrained by our observations because of the limited spatial resolution in the mid-IR, so it is taken after the near-IR interferometric constraint. Its influence nevertheless depends on the geometry of the inner wall, which is assumed to be vertical in the present modelling (see further discussion in Sect. 5.3).

3. The Silicate content. Based on the nominal MRN distribution, the silicate content was varied from 62.5 to $97 \%$. Increasing the ratio of silicates favours higher values of $\beta$ and translates the SED $\chi^{2}$-map towards larger scale heights. The combination of both results in a significant variation in $\beta$ and $h_{100}$ with maximum values of 1.33 and $10 \mathrm{AU}$, respectively.

4. The grain size. We have considered the cases of two populations of small $(a=0.01 \mu \mathrm{m})$ or large $(a=1 \mu \mathrm{m})$, pure silicate grains. The model visibilities are hardly affected by the grain size (at least in the range probed by our simulations). More noticeable is the change in the spectrum: the large-grain population tends to flatten the silicate-band emission between 10 and $13 \mu \mathrm{m}$ and yields a poorer agreement for the total fit. While the spectral constraint remains almost unchanged, it tends to favour slightly flatter disks compared to the MRN distribution or the small grains population $(\delta \beta \sim 0.025)$. Unlike the analysis proposed by Bouwman et al. (2000) and van Boekel et al. (2005), where only the optically thin upper layer of the disk was modelled with single-temperature grains, our improved disk modelling does not allow us to draw conclusions on the possible grain growth in this young system. The component of submicronic grains seems, however, to be dominant in order to best reproduce the shape of the silicate band in the $10-13 \mu \mathrm{m}$ interval.

5. The inclination. This parameter has a weak impact on the global fit as long as it remains consistent with a face-on view of the system. We also tested a much greater inclination of $65 \mathrm{deg}$, as was commonly used before the recent $\mathrm{mm}$ 
observations. The fit results mainly in a thicker scale height, which is consistent with our modelling an almost pole-on disk with a very inclined structure. The visibility constraint on the $\chi^{2}$-map is broadened. Although this inclination should be independently determined for the inner disk in the mid-IR, the value in our simulation is consistent with most of the recent high-resolution observations of this disk. In any case, it has to be lower than 70-75 deg, since the silicate-band would not appear in emission anymore for higher values.

\subsection{The paradigm of the inner rim and the possible shadowed region}

Our model is based on a simple flaring geometry with a vertical inner rim. This basic model is successful in reproducing the mid-IR continuum spectrum, as well as the spectrally-dispersed visibilities reported in this paper. To account for the large flux level observed in the near-IR ("3 $\mu$ m-bump"), Natta et al. (2001) and Dullemond et al. (2001) proposed a modified scheme for the inner rim. In this scenario, the inner wall at the dust truncation radius puffs up because it is directly illuminated by the central star, resulting in an increased gas and dust scale height compared to the Chiang \& Goldreich (1997) model (where only grazing illumination is considered in the upper disk layer). With observational and theoretical evidence, it has recently been argued that this rim might be curved as a result of the gas-density dependence of the dust-sublimation temperature (Isella \& Natta 2005) or through dust-grain growth and settling (Tannirkulam et al. 2007). The location and the shape of the rim should indeed depend on these two factors, as well as on the possible existence of a gas component (optically thin or thick) inside the dust depletion zone, as proposed by Monnier \& Millan-Gabet (2002) and Monnier et al. (2005) to account for the empirical near-IR size - stellar luminosity relation derived for disks around Herbig stars.

The curved-rim model was tentatively applied to AB Aur by Isella et al. (2006) in order to self-consistently reproduce the near-IR spectrum together with IOTA/PTI visibilities, but without success. The authors conclude that temperatures higher than $2500 \mathrm{~K}$ would be required to fit the observational data, a value much higher than the sublimation temperature of the most refractory materials. Hot gas inside the dust cavity is suspected to provide a substantial contribution to the near-IR luminosity. Its presence is attested to by recent NIRSPEC observations of $\mathrm{Br} \gamma$ and $\mathrm{CO}$ lines and is also expected to account for recent $K$-band very long baseline $(\geq 200 \mathrm{~m}$ ) interferometric measurements (Tannirkulam et al. 2008). The presence of a hot $(\sim 2400 \mathrm{~K})$, compact $(\leqslant 0.1 \mathrm{AU})$ gaseous continuum emission has been recently proven from dispersed interferometric observations at Keck (Eisner et al. 2007) around another Herbig Ae star (MWC 480), a system with an intermediate mass accretion rate ( $\dot{M}_{\text {acc }} \geq 10^{-7} M_{\odot} /$ yr). Estimations of the mass accretion rate for AB Aur vary from $\dot{M}_{\text {acc }}=3 \times 10^{-7} M_{\odot}$ (Brittain et al. 2007, based on $\operatorname{Br} \gamma$ luminosity) to $10^{-9} M_{\odot}$ (Telleschi et al. 2007, from $\mathrm{X}$ ray observations, see further discussion therein).

The presence of a compact component of hot gas in the system would affect the irradiation scheme of the inner dust wall. Together with the possible puff-up of the rim, it could also change the visibiility profile in the mid-IR, especially yielding a moderate increase in the visibility at the short-wavelength edge of the $N$-band. Although the reported MIDI visibilities are in reasonable agreement with those produced by a vertical rim model, we do not exclude the presence of a curved, puffed-up rim, eventually associated with a small extended shadowed region, but more observations (with a denser spatial frequency coverage) are needed to clearly detect such a fine morphological feature.

\section{Summary}

In the context of the MIDI GTO, we observed the thermal warm dust emission of the AB Aurigae disk. We analyse the MIDI data and the existing SED using the Monte-Carlo code MC3D. Our main results are

- The inner dust disk is optically thick at $10 \mu \mathrm{m}$. The data are well-fitted by a silicate-dominated dust mixture, the Si-band emission corresponding to $70-90 \%$ of the total flux measured by ISO, with possible hints of time variability.

- The radial structure of the inner disk is directly measured by the interferometer MIDI, since the disk is resolved on all baselines.

- Using the MIDI visibilities and the IR SED, we are able to determine the flaring index $(\beta)$ precisely and unambiguously, which is mostly constrained by the interferometric observations, as well as the inner disk scale height $\left(h_{100}\right)$, which is constrained by the SED.

- Last but not least, we also investigated the hidden dependencies (inherent in such models) associated to the scale height and its flaring index. We explored a large grid of possible values for the model input parameters. We find that, since the disk structure is resolved by the interferometer, the flaring index remains robust with respect to the other parameters, such as the dust mass, the inner radius, or the dust composition.

- The resulting disk structure is consistent with a flared inner disk with $\beta$ in the range $1.25-1.30$ (for $r \lesssim 10 \mathrm{AU}$ ), in agreement with values derived from theoretical models developed by D'Alessio et al. (1999). This result confirms by direct imaging that $\mathrm{AB}$ Aur belongs to the Group I sources of the Meeus et al. (2001) classification and sets new stringent constraints on the disk morphology in the planet-forming regions.

Acknowledgements. A. Dutrey acknowledges the MIDI Paranal staff for the help provided during the observations, with particular thanks to S. Morel and Ch. Hummel. S. Wolf and A. Schegerer were supported by the German Research Foundation (DFG) through the Emmy Noether grant WO 857/2. A. Dutrey and E. Di Folco were partially supported by the "Programme National de Physique Stellaire". E. Di Folco also thanks the Fizeau exchange foreign visitor programme.

\section{References}

Artymowicz, P., Clarke, C. J., Lubow, S. H., \& Pringle, J. E. 1991, ApJ, 370, L35

Bouwman, J., de Koter, A., van den Ancker, M. E., \& Waters, L. B. F. M. 2000, A\&A, 360, 213

Brittain, S. D., Simon, T., Najita, J. R., \& Rettig, T. W. 2007, ApJ, 659, 685

Chen, C. H., \& Jura, M. 2003, ApJ, 591, 267

Chiang, E. I., \& Goldreich, P. 1997, ApJ, 490, 368

Corder, S., Eisner, J., \& Sargent, A. 2005, ApJ, 622, L133

D’Alessio, P., Canto, J., Calvet, N., \& Lizano, S. 1998, ApJ, 500, 411

D’Alessio, P., Calvet, N., Hartmann, L., Lizano, S., \& Cantó, J. 1999, ApJ, 527, 893

Dullemond, C. P., Dominik, C., \& Natta, A. 2001, ApJ, 560, 957

Eisner, J. A., Lane, B. F., Hillenbrand, L. A., Akeson, R. L., \& Sargent, A. I. 2004, ApJ, 613, 1049

Eisner, J. A., Chiang, E. I., Lane, B. F., \& Akeson, R. L. 2007, ApJ, 657, 347

Fukagawa, M., Hayashi, M., Tamura, M., et al. 2004, ApJ, 605, L53

Grady, C. A., Woodgate, B., Bruhweiler, F. C., et al. 1999, ApJ, 523, L151

Hernández, J., Calvet, N., Briceño, C., Hartmann, L., \& Berlind, P. 2004, AJ, 127,1682 
Isella, A. \& Natta, A. 2005, A\&A, 438, 899

Isella, A., Testi, L., \& Natta, A. 2006, A\&A, 451, 951

Kenyon, S. J., \& Hartmann, L. 1987, ApJ, 323, 714

Kessler, M. F. 2000, ISA Trans., 29

Leinert, C., van Boekel, R., Waters, L. B. F. M., et al. 2004, A\&A, 423, 537

Lin, S.-Y., Ohashi, N., Lim, J., et al. 2006, ApJ, 645, 1297

Liu, W. M., Hinz, P. M., Hoffmann, W. F., et al. 2005, ApJ, 618, L133

Mannings, V., \& Sargent, A. I. 1997, ApJ, 490, 792

Mariñas, N., Telesco, C. M., Fisher, R. S., Packham, C., \& Radomski, J. T. 2006, ApJ, 653, 1353

Mathis, J. S., Rumpl, W., \& Nordsieck, K. H. 1977, ApJ, 217, 425

Meeus, G., Waters, L. B. F. M., Bouwman, J., et al. 2001, A\&A, 365, 476

Millan-Gabet, R., Schloerb, F. P., \& Traub, W. A. 2001, ApJ, 546, 358

Millan-Gabet, R., Monnier, J. D., Berger, J.-P., et al. 2006, ApJ, 645, L77

Monnier, J. D., \& Millan-Gabet, R. 2002, ApJ, 579, 694

Monnier, J. D., Millan-Gabet, R., Billmeier, R., et al. 2005, ApJ, 624, 832

Natta, A., Prusti, T., Neri, R., et al. 2001, A\&A, 371, 186

Oppenheimer, B. R., Brenner, D., Hinkley, S., et al. 2008, ApJ, 679, 1574

Pantin, E., Bouwman, J., \& Lagage, P. O. 2005, A\&A, 437, 525

Piétu, V., Guilloteau, S., \& Dutrey, A. 2005, A\&A, 443, 945
Piétu, V., Dutrey, A., Guilloteau, S., Chapillon, E., \& Pety, J. 2006, A\&A, 460, L43

Pontefract, M., Drew, J. E., Harries, T. J., \& Oudmaijer, R. D. 2000, MNRAS, 319, L19

Rodríguez, L. F., Zapata, L., \& Ho, P. T. P. 2007, Rev. Mex. Astron. Astrofis., 43, 149

Semenov, D., Pavlyuchenkov, Y., Schreyer, K., et al. 2005, ApJ, 621, 853

Sloan, G. C., Kraemer, K. E., \& Price, S. D. 2003, in The Calibration Legacy of the ISO Mission, ed. L. Metcalfe, A. Salama, S. B. Peschke, \& M. F. Kessler, ESA SP, 481, 447

Tannirkulam, A., Harries, T. J., \& Monnier, J. D. 2007, ApJ, 661, 374

Tannirkulam, A., Monnier, J. D., Millan-Gabet, R., et al. 2008, ApJ, 677, L51

Telleschi, A., Güdel, M., Briggs, K. R., et al. 2007, A\&A, 468, 541

van Boekel, R., Min, M., Waters, L. B. F. M., et al. 2005, A\&A, 437, 189

van den Ancker, M. E., de Winter, D., \& Tjin A Djie, H. R. E. 1998, A\&A, 330, 145

Weingartner, J. C., \& Draine, B. T. 2001, ApJ, 548, 296

Wolf, S. 2003, Computer Physics Communications, 150, 99

Wolf, S., Henning, T., \& Stecklum, B. 1999, A\&A, 349, 839

Wolf, S., Padgett, D. L., \& Stapelfeldt, K. R. 2003, ApJ, 588, 373

Wood, K., Wolff, M. J., Bjorkman, J. E., \& Whitney, B. 2002, ApJ, 564, 887 